\title{
Téoros
}

Revue de recherche en tourisme

\section{Contre-urbanisation et résidences secondaires}

\section{Marcel Samson}

Volume 2, numéro 3, octobre 1983

Les multiples facettes de l'aménagement touristique

URI : https://id.erudit.org/iderudit/1080825ar

DOI : https://doi.org/10.7202/1080825ar

Aller au sommaire du numéro

Éditeur(s)

Université du Québec à Montréal

ISSN

0712-8657 (imprimé)

1923-2705 (numérique)

Découvrir la revue

Citer cet article

Samson, M. (1983). Contre-urbanisation et résidences secondaires. Téoros, 2(3),

13-16. https://doi.org/10.7202/1080825ar d'utilisation que vous pouvez consulter en ligne.

https://apropos.erudit.org/fr/usagers/politique-dutilisation/ 


\title{
Contre-urbanisation et résidences secondaires
}

\author{
Par Marcel Samson
}

Comme dans la plupart des pays industrialisés, le Canada (et le Québecl a connu ces dernières années un renversement des tendances démographiques séculaires ${ }^{~(1)}$. En effet, les courants migratoires qui ont transformé un Québec rural en un Québec urbain ne se font plus sentir depuis le début des années 70 . Ce retournement est particulièrement spectaculaire puisque la tendance que les demographes avaient observée au recensement de 1976 s'est poursuivie au recensement de 1981 de façon encore plus marquée.

"Le recensement de 1976 a en effet révélé que, pour la première fois dans /histoire canadienne les régions rurales ont connu (entre 1971 et 1976 ) un taux d'accroissement de la population plus elevé que les régions urbaines, et la difference entre les deux taux et encore plus grande entre 1976 et $7987^{\circ} \cdot 2$

Nous sommes en présence d'un phènomène qui se distingue de la rurbanisation ${ }^{3}$ en ce sens que ce n'est pas seulement à des mouvements migratoires de population urbaine vers les zones rurales auxquels on assiste mais egalement à une croissance de population plus rapide en zone rurale qu'en zone urbaine ${ }^{|4|}$. C'est ce que Brian Berry et d'autres ont appelé la contre-urbanisation ${ }^{|5|}$. A la différence de l'urbanisation, ce proces sus implique en principe une décroissance de la taille, de la densité et de l'hétérogénéité en mëme temps qu' une déconcentration démographique. Cependant, it se rapproche de l'urbanisation par certaines manifestations spatiales similaires (comme la rurbanisation): solde migratoire positif, proportion décroissante d'agriculteurs, transforma tion du marché foncier et constructions nouvelles ${ }^{(6)}$. Pour eviter toute confusion dans les termes, il est important aussi de souligner que la contre-urbanisation ou la rurbanisation sont des phénomènes diffé rents de la péri-urbanisation parce qu'ils ne constituent pas une extension contigüe à l'agglomeration urbaine.

Au Québec, les premières observations sur l'importance de l'exode urbain ont démon- tré qu'en moyenne $17,5 \%$ de la population des municipalités rurales nonmetropolitaines habitait en ville en $1971^{(7)}$ Le géographe Brunet soulignait alors qu'en direction nord de l'agglomération de Montréal "Vinfluence métropolitaine se fait sen. tir p/us loin et avec p/us d'intensité qu'au sud, en particuler par l'intermediaire des migrations hebdomadaires et saisonnieres de villegiature des Montréalais vers une multitude de résidences secondaires" " 18 ". Des chercheurs de I'O.P.D.O. avaient déjà émis l'hypothèse que la localisation des zones d'accroissement démographique au nord de Sainte-Scholastique et SaintJérôme "s"expliquerait pour une grande part grâce au phénomene de villegiature, $c$ 'est-a-dire fixation d'une population nouvelle, principalement citadine, par la transformation de résidences secondaires en résidences permanentes et par l'offre de services touristiques"s 1 !

A partir de ces hypotheses, nous avons voulu, par une étude exploratoire, vérifier l'importance de la résidence secondaire dans le processus de la contre-urbanisation dans la région administrative de Montréal. Pour ce faire, nous avions conçu deux brefs questionnaires: un premier, par entrevue

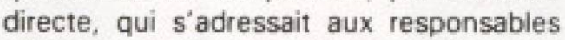
municipaux des quelques municipalités sélectionnées et un deuxième questionnaire, postal celui-ci, qui s'adressait à un petit échantillon de ménages d'origine urbaine néo-résidents permanents de ces mémes municipalités.

\section{Les municipalités d'accueil}

Pour circonscrire les municipalités où le phé nomène de contre-urbanisation pouvait jouer, nous avons utilise trois criteres: le critère de croissance démographique d'abord, ensuite un critère de présence majeure de la villegiature (résidences secondaires) dans une municipalité et, enfin, un troisième critère qui consistait en ce que la municipalite ait acquis entre 1971-1976 une proportion minimale d'ex-Montréalais irégion métropolitainel immigrants, soit $40 \%$ ou plus ${ }^{11}$.
Dans un premier temps, seules les municipalites ayant eu une croissance en chiffre absolu de 200 habitants et plus ont été retenues; ce qui a permis d'exclure des municipalités qui auraient obtenu un pourcentage excessif de $100 \%$ de croissance en passant de 100 habitants à 200 habitants. Nous avons ensuite utilisé, pour le deuxième critère, les résultats de l'étude de I'O.P.D.O sur la récréation de plein-air ${ }^{1121}$. A partir d'un fichier des résidences secondaires constitué par le service de facturation de l'Hydro-Québec, les auteurs ont établi la fonction résidentielle de villegiature ${ }^{113}$. Nous avons pris comme base un indice égal à 100 , c'est-à-dire que le nombre de residences secondaires est au moins egal au nombre de résidences principales.

En ajoutant le troisième critère, celui d'une proportion minimale d'ex-Montréalais, nous avons retenu 22 municipalités. Pour les cal culs de croissance démographique, nous avons dú regrouper certaines municipalites à cause des changements de territoire sur venus dans les années précédentes.

Parmi ce groupe de 22 municipalités, nous avons éliminé la municipalité de Mille-lsles à cause de son trop petit nombre d'habitants. A cause d'impondérables quatre municipalites n'ont pu être visitees iSte Agathe, St-Colomban, Ste-Mélanie et St Paul-de l"ile-aux-Noix). Nous avons rencontré les responsables municipaux des 17 autres municipalités. II est à remarquer qu'une seule municipalité dans la région sud (St-Paul-de-17ile-aux-Noix) de Montréal répondait aux trois critères. Cela n'est pas surpre nant puisque, comme l'affirmait le geogra phe Brunet. l'exode urbain d'origine métropolitaine privilégie la direction nord de l'agglomeration ${ }^{\text {I4). }}$, ce dernier critère com. biné aux deux autres avaient éliminé toutes les municipalités de la région sud.

Portrait sommaire

des municipalités:

amenagement du territoire et niveau des services municipaux

Au printemps ' 82 la plupart des 17 munici palités retenues pour étude ne faisaient pas 
Tableau 1

Municipalités retenues à partir des critères de croissance, de fonction de villégiature et de minimum d'ex-Montréalais

\begin{tabular}{|c|c|c|c|c|c|c|}
\hline \multirow[b]{2}{*}{ Municipalités } & \multirow[b]{2}{*}{$\begin{array}{c}\text { Pourcentage } \\
\text { d'ex-Montráalais } \\
\text { 1971-1976 }\end{array}$} & \multirow[b]{2}{*}{$\begin{array}{l}\text { Nombre } \\
\text { résidences } \\
\text { secondaires }\end{array}$} & \multirow[b]{2}{*}{$\begin{array}{l}\text { Fonction de } \\
\text { villégiature }\end{array}$} & \multicolumn{3}{|c|}{ Croissance démographique } \\
\hline & & & & 1971.1976 & $1976-1981$ & $\begin{array}{r}\text { Population } \\
\text { totale } \\
1981\end{array}$ \\
\hline Chertsey 6 Entrelacs & $63,3681,5$ & 2898 & 556 & 29,0 & 22.4 & 2067 \\
\hline Lac Superieur \& Val-des-Lacs & $67,8 \& 92,0$ & 790 & 261 & 21,7 & 27,1 & 1120 \\
\hline Mille isies & 66,1 & 340 & 197 & 17,0 & 47,1 & 637 \\
\hline Morin-Heights & 69,0 & 668 & 136 & 3,6 & 13,0 & 1540 \\
\hline St-Adolphe d'Howard & 40,0 & 2161 & 520 & 1,4 & 22,6 & 1656 \\
\hline Ste-Agahe & 66.7 & 1222 & 399 & 34,3 & 42,7 & 1132 \\
\hline Ste-Agathe-Sud & 43,3 & 575 & 201 & 18,8 & 26,2 & 1333 \\
\hline St-Alphonse-de-Rodriguez & 77,8 & 1557 & 464 & 25,7 & 30.9 & 1402 \\
\hline Ste-Anne-des-Lacs & 84,2 & 555 & 211 & 40,2 & 24.6 & 974 \\
\hline St-Calixte \& St-Hippolyte & $74,0 \& 65,2$ & 5572 & 135 & 30,6 & 42,4 & 6113 \\
\hline St-Colomban & 64.6 & 835 & 151 & 64,8 & 28.4 & 2263 \\
\hline St-Jean-de-Matha & 72,5 & 1171 & 203 & 10,3 & 22,3 & 2483 \\
\hline Ste-Lucie-des-Laurentides $\Theta$ Val-David & $42,6 \& 51,4$ & 1610 & 188 & 24,4 & 17,0 & 3010 \\
\hline Ste-Marguerite-du-Lac-Masson & 53,1 & 1998 & 486 & 15,0 & 8,7 & 1712 \\
\hline Ste-Mélanie & 39.1 & 455 & 149 & 7,6 & 47,4 & 1580 \\
\hline St-Paul de l'lle-aux-Noix & 61,4 & 564 & 163 & 14,8 & 25,9 & 1379 \\
\hline St-Sauveur & 67,1 & 1221 & 321 & 22,8 & 34,6 & 1425 \\
\hline Val-Morin & 56,9 & 996 & 276 & 23.7 & 15,1 & 1280 \\
\hline
\end{tabular}

encore partie d'une municipalité régionale de comté (MRC) mais étaient sur le point d'y adhérer; pour les autres cette régionalisation était toute récente. II faut donc interpréter nos informations dans ce contexte.

De façon générale, on constate que ces municipalités de villégiature possèdent des caractéristiques semblables: statut juridique de paroisse ou de canton, faible densité de I'habitat par rapport a la superficie du territoire (variant de $20 \mathrm{~km}^{2}$ a $300 \mathrm{~km}^{2}$ ), développement anarchique avec peu ou pas de réglementations ou encore une applicaion très large de la réglementation existante.
La forme spatiale qu'ont prise ces agglome rations se ressemble enormement. Du centre du village traditionnel où sont regroupés les équipements publics ou communautaires (église, hôtel de ville, ecole,.... let les com. merces partent des routes qui mènent aux résidences secondaires ou principales qui s'étalent en chapelet sur les rives des lacs du territoire.

Avant 1970, seulement 5 municipalités sut 17 disposaient de règlements de zonage et deux seulement possedaient un plan directeur d'urbanisme avant cette même date. Même les règlements régissant la construc-

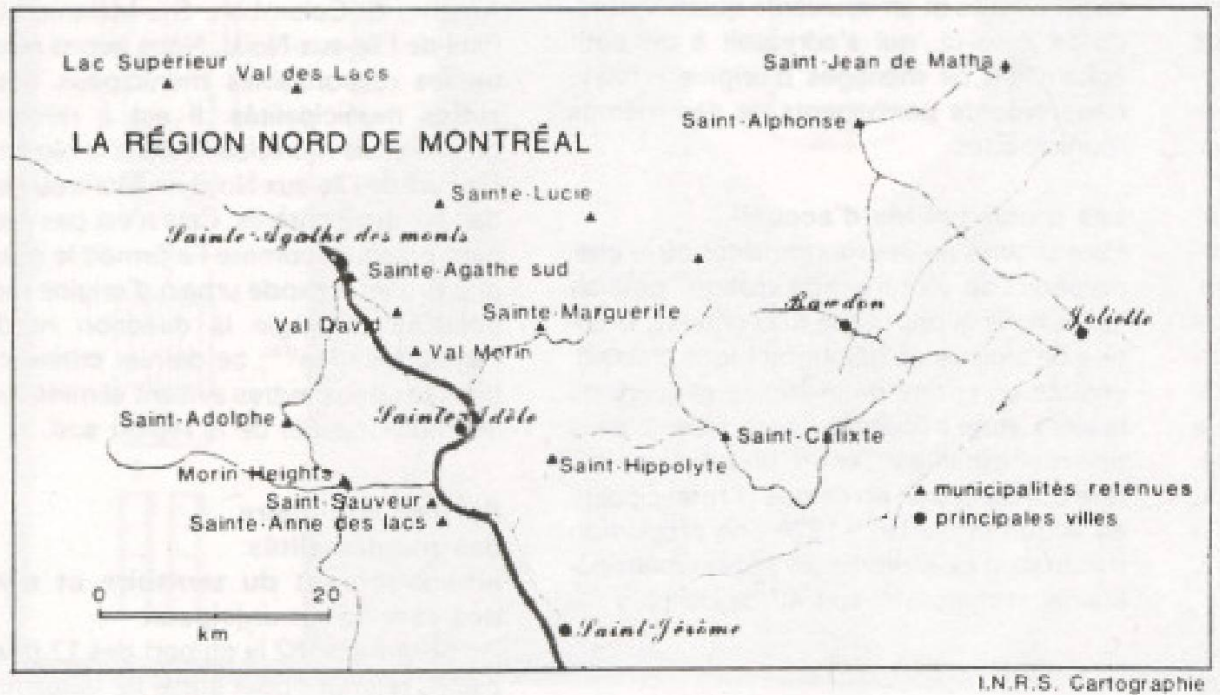

tion ne sont pas apparus avant 1970 pour près de la moitié d'entre elles. Par inventaire rapide des battiments existants et de l'aveu même des responsables municipaux, il est évident que l'ensemble de la réglementation en vigueur à une époque ou à une autre a été l'objet d'interprétations parfois fort fantaisistes ou n'a tout simplement pas eté appliqué. II est bien connu par exemple que dans plusieurs municipalités on a fait bon marché des permis de construction jusque dans les année "70.

Quant au zonage agricole, seule St-Jean de Matha a été touchée alors que la Commission de protection du territoire agricole decrétait en juin 1981 que $70 \%$ du territoire de cette municipalité tombait sous ce zonage. Ce qui n'a pas eu l'heur de plaire aux élus municipaux qui voyaient là un frein aux activités de construction. En fait, de par sa localisaiton, dans la région de Lanaudière. St-Jean de Matha conserve une vocation agricole aussi bien que touristique alors que les municipalités localisées dans le corridor des Laurentides ont une vocation presqu'exclusivement touristique.

En ce qui concerne l'aménagement des rives de lacs comme telles, il est révélateur que seules six municipalités possédaient jusqu' au moment de l'étude une plage publique. Cela dénote certainement une absence de contrôle public sur le développement du territoire dans les années antérieures.

L'entretien des chemins et la cueillette hebdomadaire des ordures ménagères sont habituellement les seuls services qui sont offerts à l'ensemble de la population, aussi 
bien résidents principaux que résidents secondaires, comme c'est le cas dans pres. que toutes les régions rurales du Québec. Les infrastructures d'égout et d'aqueduc existant dans la plupart des municipalités ne desservent qu'une faible proportion du territoire $(5$ a $20 \%)$, generalement dans le noyau central de l'agglomeration.

Les résidents permanents et les villégiateurs qui ne sont pas desservis doivent se doter de puits artésiens pour l'approvisionnement en eau et d'installation septiques pour l'éva: cuation des eaux usées. Sur ce dernier point, il est intéressant de noter que l'action du ministère de l'Environnement aura un impact important sur les développements futurs de l'ensemble des municipalités. Le decret sur le traitement des eaux usées qui s'applique depuis le 1er janvier 1983 a l'ensemble des municipalités du Québec signifie en pratique qu'on exigera pour les nouveaux développements des lots de plus de $4000 \mathrm{~m}^{2}$ en bordure des lacs et de plus de $3000 \mathrm{~m}^{2}$ en deuxième rangée.

Cette législation appréciable du point de vue de l'environnement signifiera cependant en pratique pour ces municipalités que la construction de résidences secondaires devrait connaitre des coûts plus élevés alors mème qu'elle avait ralenti à cause de la crise inflationniste. II faut penser en tous les cas que la construction nouvelle de la residence secondaire utilisée sur une base annuelle ou saisonnière deviendra de plus en plus à la portee des categories supérieures de revenus.

\section{Croissance démographique et}

\section{contre-urbanisation}

Avec les données du Tableau 1, on peut observer la croissance démographique de chacune des municipalités retenues entre 1971 et 1981 . A ce niveau micro-spatial, it faut bien sür s'arrêter aux grandes tendances générales; cependant, il est possible d'indiquer certains facteurs qui au niveau local ont pu influencer cette croissance.

II faut remarquer tout d'abord que l'apport d'une population nouvelle d'origine métropolitaine a été très important pour l'ensemble des municipalités entre 1971 et 1976 . Nous l'avons dejà mentionné, les chiftres des ex-urbains entre 1976-1981 n'étaient pas disponibles au moment de l'étude mais nous n'avons aucune raison de croire que l'apport d'ex-Montrealais est plus faible dans la croissance démographique durant cette perriode. Par ailleurs, la croissance naturelle, on le sait, n'a eu aucune influence significative. C'est donc par un solde migratoire positif que l'on peut expliquer la croissance des dernières annees. Dans quelques cas, cette croissance a benéficié d'un changement de limite territoriale de quelques municipalités mais cela n'a pas été un facteur important.
Nous avons voulu savoir comment cette croissance démographique s'était refletee au niveau des activités de construction. Les données que nous avons pu obtenir reflètent dans la mesure du possible la realité. Nous ne sommes pas sans savoir qu'un bon nombre de réparations ou de rénovations domiciliaires se font sans permis de cons: truire dans les municipalités rurales, particulièrement dans les zones de villegiature Pis encore, dans quelques municipalités, peu nombreuses heureusement, il taut deplorer le manque de rigueur dans les compilations de permis de construction quand ce n'est pas une absence même de compilation pour les années antérieures à 1980.

Les chiffres qui nous ont été transmis montrent tout de mème que l'activité de cons. truction (nouvelle ou rénovations diverses) a été relativement soutenue depuis 1976 mème si la construction de nouvelles résidences a connu une chute importante dans certaines municipalités.

De l'ensemble des donnees, nous pouvons faire quelques hypothèses que devraient confirmer des recherches plus poussées:

Plus loin on pénètre vers le nord, plus la construction de résidences secondaires augmente par rapport à la construction de résidences permanentes.

A l'inverse, les municipalités qui sont situées plus près de la région métropolitaine et qui bénéficient d'accès routiers faciles (dans la région de Lanaudière ou dans les Laurentides) ont connu une activité de construction qui reflète certains aspects du processus de contreurbanisation.

La conversion de résidences secondaires en résidences principales est un phé nomène présent avec plus ou moins d'intensité dans toutes les municipalités retenues. Cette conver sion est entrainee par des facteurs divers comme la proximité et le niveau des équipements publics, la proximité des voies de communication, le niveau de taxation municipale, la qua lité de I'environnement iplan d'eau. boise, montagnes, etc...l. le type et le niveau de vie moyen de la population de résidents secondaires, la composition et l'âge des ménages,...

Le stock de "chalets" Irésidence secondaire d'étell a tendance à augmenter avec la distance de la région métropolitaine. De mème, à cause de la configuration spatiale, de la vocation du territoire ainsi que pour des raisons historiques, la proportion de chalets a tendance à ètre plus élevée dans la région de Lanaudière plu. tôt que dans la region des Laurentides.
Qui sont ces nouveaux immigrants? Dans les limites de cette étude et à l'aide de nos informateurs privilégiés, nous avons constitué un petit échantillon de 108 ména ges à qui nous avons adressé un questionnaire par la poste. Considérant l'instrument utilise, nous avons reçu un taux favorable de 52 questionnaires complétès. Six questionnaires ont été annulés puisque les répon dants n'étaient pas des ex-urbains. Nous avons done utilise 46 questionnaires à partir desquels nous avons tenté d'avoir une première idee du type de population qui a effectué ce changement résidentiel et dans quelles conditions générales cela s'est fait.

A la lecture de certaines variables comme l'occupation, la scolarisation et le revenu, nous nous apercevons qu'un niveau socioeconomique élevé n'est pas nécessairement le critère le plus important qui explique le choix qui a été fait par ces nouveaux rési. dents permanents.

Ainsi, les données sur le revenu familial brut par exemple nous révèlent que 24 répondants sur 46 déclarent des revenus de moins de 20000 s tandis que 8 autres ont des reve nus qui se situent entre $20000 \$$ et $30000 \$$ Pour la variable scolarisation, 31 répondants ont atteint les niveaux primaire et secondaire et représentent donc une forte majorité de notre petit echantillon.

Nous croyons que c'est plutōt au niveau des occupations que se trouve une des raisons de ce changement résidentiel. II nous faut d'abord distinguer deux types de nouveaux résidents permanents. les retraites et les actifs. Dans notre échantillon, nous retrou. vons 17 répondants qui se déclarent a la retraite. Cet établissement à la campagne de retraités d'origine urbaine a des rapports évidents avec le phénomène de résidences secondaires. II ne faut pas confondre ce mouvement avec celui, encore mal connu ici, du retour au "coin de terre natal" des émigrés d'origine rurale ayant passé en ville leur vie active. Le deuxieme groupe, celui des actifs, est compose de personnes qui ont des occupations fort diverses, de postes de direction jusqu'aux emplois dans le secteur des services et de la construction. On peut presumer que, parmi les actifs, ceux qui travaillent en ville ont une assez grande maitrise de leur temps de travail; ce facteur parmi d'autres peut expliquer ce changement résidentiel. Près des $2 / 3$ des actifs ont affirmé que leur lieu de travail était localisé à Montreal ou dans sa banlieue; ce qui équivaut pour la majorité de ces derniers à une distance-temps variant de 45 à 60 minutes entre leur domicile et leur lieu de travail.

\section{Dynamique de la conversion}

Environ $75 \%$ des répondants ont affirmé que leur changement résidentiel avait entrainé des travaux de rénowation lisolation particulièrement) à leur résidence actuelle. Pour la moitié d'entre eux qui possedaient une résidence secondaire d'été (un chalet), 
cela s'est traduit par des transformations plus importantes lagrandissement, murs extérieurs, salle d'eau, cuisine, etc...) que pour les 8 personnes qui ont affirmé avoir procédé à quelques amélior ations; 15 autres répondants ont bènéficié d'une construction neuve.

Pour la majorite des menages, le changement résidentiel s'est accompli dans les dix dernières années; pour 31 répondants cela ne remonte méme pas à plus de 5 ans. Cette émigration de la ville à la campagne constitue donc pour les ménages de notre échantillon un fait relativement récent. Cela correspond aussi aux données que nous avons sur la croissance démographique qu'ont connue les municipalités de villégiature depuis 1971. II est aussi intéressant de savoir que 23 nouveaux résidents étaient propriétaires d'une résidence secondaire depuis plus de 5 ans avant d'effectuer cette conversion et cela tend a confirmer que la contre-urbanisation par l'effet de la résidence secondaire est un phénomène nouveau.

\section{Conclusion}

La discussion que nous avons eue sur l'impact de la résidence secondaire sur la contreurbanisation soulève beaucoup de questions auxquelles nous n'aurions su répondre dans cet article. Nous n'avons que commencé à explorer le phénomène de contreurbanisation dans les municipalités de villégiature.

Selon l'étude de Termote et Mongeau, "/a contre-urbanisation existe au Québec, mais elle se localise essentiellement, quoique de moins en moins, dans les régions adjacentes aux grands centres urbains. Elle touche en fait très peu les régions éloignées des grands centres, et elle se manifeste surtout dans les comtés deja fortement urbanisés" ${ }^{\text {15! }}$. Les tendances qu'on a pú observer dans les régions des Laurentides et de Lanaudière viennent appuyer cette affirmation.

Mais alors que le phénomène de contreurbanisation est apparu surtout pour des raisons economiques semble-t-il itel est en tout cas le diagnostic que certains auteurs font pour les Etats-Unisi, les facteurs qui concourent à ce mème processus dans les municipalités de villégiature relèvent plus de ce qu'on appelle la qualité de la vie' 116 .

La contre-urbanisation, un phénomène temporaire? Les problème économiques, le coút de l'essence par exemple, peuvent-ils freiner cette tendance? A première vue, c'est un changement migratoire qui semble avoir des bases solides et qui pourrait bien se poursuivre; c'est un phénomène dont les aspects restent a approfondir.

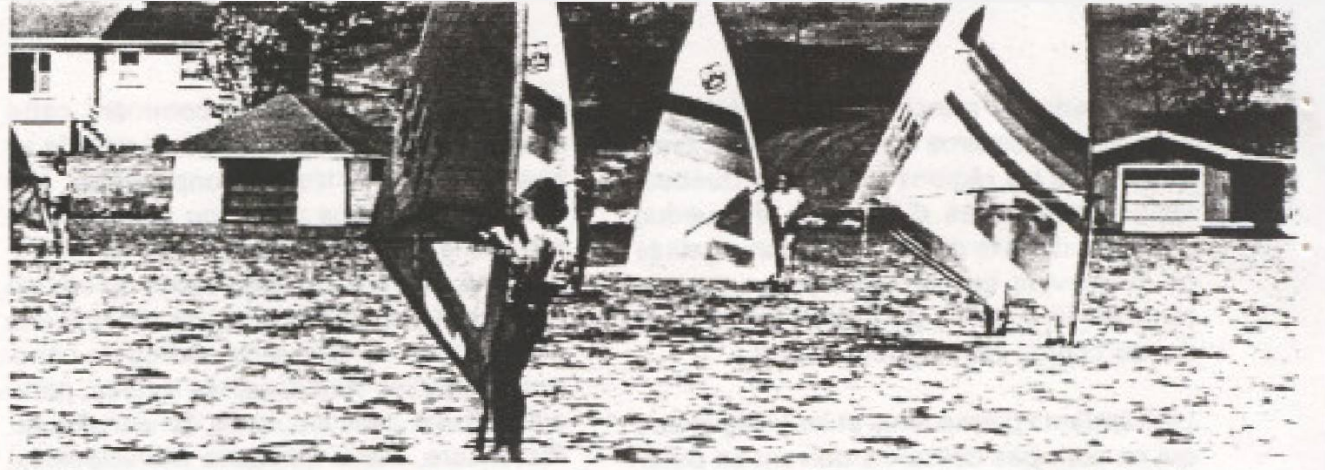

Les facteurs qui concourent au processus de contre-urbanisation dans les municipalités de villégiature relèvent plus de ce qu'on appelle la qualité de vie.

Appendice:

Problème de fiabilité des données sur la résidence secondaire

\section{Statistiques generales}

Le recensement de 1971 a été le premier et le seul à inclure une question sur la résidence secondaire à partir de laquelle plusieurs informations sur les ménages propriétaires ont été obtenues ${ }^{177}$. Depuis lors, seules des enquêtes sporadiques sur divers aspects du tourisme ou du loisir ont apporté des informations plus récentes et aussi détaillées sur ce type d'habitat et sur les ménages-propriétaires.

II existe aussi un sondage annuel effectué par Statistique Canada auprès d'un échan. tillon de ménages et qui porte sur l'équipement meenager; les résultats sont publiés par province $^{1180 .}$. Mais les données de ce dernier sondage se limitent au nombre de "maisons de villégiature" par ménage. A ce niveau, il existe dejà des problemes de concordance de données. Au recensement de 1971, on obtenait un taux de $8,6 \%$ de ménages: propriétaires de résidences secondaires pour I'ensemble de la province. Dans le sondage annuel de 1981 , ce taux s'établit à $8 \%$ approximativement, ce qui signifie qu'il $y$ aurait encore moins de propriétaires qu'en 1971; cela nous semble aberrant. D'autant plus que les estimations à partir de différentes enquêtes établissent cette proportion autour de 14 ou $15 \%$ et même plus ${ }^{199}$. II y a peut-être là un problème de sémantique autour des termes de "résidence secondaire", "maison de villegiature" et "chalet".

\section{Statistiques locales}

Nous avons déjà abordè au cours de l'article les problèmes de statistiques au niveau local; les données tirées du róle d'évaluation des municipalites sont parfois incomplètes ou partielles de sorte que les analyses com. paratives doivent être abordées avec beau. coup de prudence. Ainsi en est-ill de l'interprétation qu'on en fait. Par exemple, un document récent de la FAPEL indiquait que le nombre de chalets dénombrés par municipalité comprenait uniquement les résiden. ces d'été. II aurait été plus juste de souligner que le rôle d'évaluation dans certaines municipalités ne comprenait que les résidences d'été tandis que dans d'autres le rôle d'éva luation comprenait à la fois les résidences d'étè et les résidences à l'année. Si tel n'était pas le cas, le Québec atteindrait un taux record de résidences secondaires par ménage. $f$

\section{Références}

(1) La recherche qui a donne lieu a ce texte a elte subventionne par les fonds F.C.A.C. du ministère de I'Education du Quebec.

(2) TERMOTE, Marc G. et Joel MONGEAU, "L ampleur de la contre urbanisation au Quebec ". Texte mod. fie d'une communication presentes au Colloque sur l'exode urbaine. Departement de Geographie. Universte de Montreal, avril 1962

(3) BAUER, G et J.M. ROUX, La rurbanisation ou la ville eparpilles. Paris. Ed. du Seuil, 1976

(4) Pour tutre considere comme "urbain" en 1976 et en 1981, un territaire doit présenter une densite de 1 000 hab. au mille carre 1400 hab. au $\mathrm{km}$ cand, en mème temps qu'un minimum de 1000 habitants En 1971. cette définition etai? plus large en termes de superficie.

5) BERRY, Brian, J.L. Urbanisation and Counfer Urbanisation. Vol. II, Urban Affairs Annual Review, Beverly Hills, California, Sage Publications Lid. 1976

(6) BAUER, G. et J.M. ROUX, op cit, D. 13

(7) BRUNET, Yves. L'exode urbain dans la region de Monveal. Notes et Documents no 80-05. Departement de Geographic. Universilé de Montreal, 1960, p. 11

(B) BRUNET, $\gamma$, op eit. p. 9.

(9) FAUTEUX, Martial et al, Notes concernant la crois: sance rbcente de l'arrière-pays et la diffusion de fur banisation dans la region montrealaise, Montréal, O.P.D.0. $1977,0.3-4$
(10) Mme Sylvie Part. assistante de rechenche a III.N.R.S Urbanisation a accompli le trayal denquéte sur lie terrain el a collabore a l'analyse de meme que Joel Mongeau, dernographe.

(11) Les compilations du recensement de 1981 sur ce point rietant pas disponibles à ce moment

(12) LABONTE, P. et F, DUMONT, La reeréation de plein-air, Dossiers techniques de la Region de Montreal, Montreal, O.P.D. O, 1980.

(13) L'indice de fonction résidentielle de willegiature se definat comme suit:

Le nombre de residences secondaires

$$
\text { Le nombre de résidences principales } \times 100
$$

Le nombre de résidences principales

(14) BRUNET. Yves op. cit., p. 14

(15) TERMOTE, M. et Joel MONGEAU, op, cit, p 22

(16) LONG, Lany. DE ARE. Diana, The Showng of Urbanisation in the U.S. Scientific American. vol. 249 , No. 1. July 1983. p. 33-41

(17) Statistique Canada, recensement de 1971 , catalogue 73711

(18) Statistique Canads, L'equipement menager, cat. 64-202. mai 1982

(19) Par exemple. Tetude de P. CLUZEAu et G. MEUNIER sur ie comportement des tamilles quebecoises en matiere de voyages de vacances en 1979 Dossier technique no 3. M.L. . . octobre 1980 